Radial and Nonradial Pulsations as Probes of Stellar Physics

ASP Conference Series, Vol. 259, 2002

C. Aerts, T.R. Bedding, \& J. Christensen-Dalsgaard, eds.

\title{
Complex Pulsation-Like Variability of the LBV Star v532 in M33
}

\author{
O. Sholukhova ${ }^{1}$, A. Zharova ${ }^{2}$, S. Fabrika ${ }^{1}$, D. Malinovskii ${ }^{1}$ \\ ${ }^{1}$ Special Astrophysical Observatory, Russia; ${ }^{2}$ Sternberg Astronomical \\ Institute, Russia
}

\begin{abstract}
We present an analysis of a light curve of LBV star v532 (Romano's star) in M33. The data consist of 881 individual photographic observations from 1960 to 2000 . The star shows extremely rich periodic variations. We find that the amplitudes of the periods depend on brightness, as they rise when the star becomes brighter. We suggest that the 13.08-day period corresponds to the star's rotation, other short periods ( $\sim 20-30$ days $)$ represent the star's pulsations, and the longer periods are instability driven wind pulsations. The spectral variability of v532 is also strong, with the spectrum excitation depending on the stellar brightness.
\end{abstract}

\section{Observations}

We study a light curve of the LBV star v532 (Romano's star) in M33. The data consist of photographic observations: the original data by Romano (1978) which were obtained from 1960 to 1977, Bulgarian Rozhen data (Kurtev et al., 2001) obtained from 1982 to 1990, Sternberg Astronomical Instutute (SAI) and Baldone observations (Sharov, 1990) obtained from 1972 to 1988 and additional new SAI data. The last observations were obtained from 1973 to 2000 with 0.5 and $0.4-\mathrm{m}$ telescopes of SAI Crimean Laboratory. There are 881 individual photographic observations in total from 1960 to 2000 . All the data were reduced to standard Johnson $B$ band. A typical accuracy of individual measurement is $0 \mathrm{~m} 1$, but it is bigger and reaches 0.2 when the star is faint. To study periodic variations we selected time intervals as shown in Fig. 1. They are Min1 (5605 days, JD 2441919 - 2447524), Min2 (1903 days, JD 2449950 - 2451853), Max (3692 days, JD 2447234 - 24450926), MaxSAI (2182 days, JD 2447740 2449922). The MaxSAI interval is homogeneous both in data (only the Sternberg Astronomical Institute data) and in stellar brightness, but it is too short for long periods analysis. We have introduced the Max interval to study long periods in maximum.

\section{Results}

We studied variability in high and low states separately. We isolated at least 20 periods from 3 days to global 4000 days variations. The majority of the periods appear only in high or low states, but some periods are stable in all states. One of them is 190 days. We find that the amplitudes of the periods depend on the 


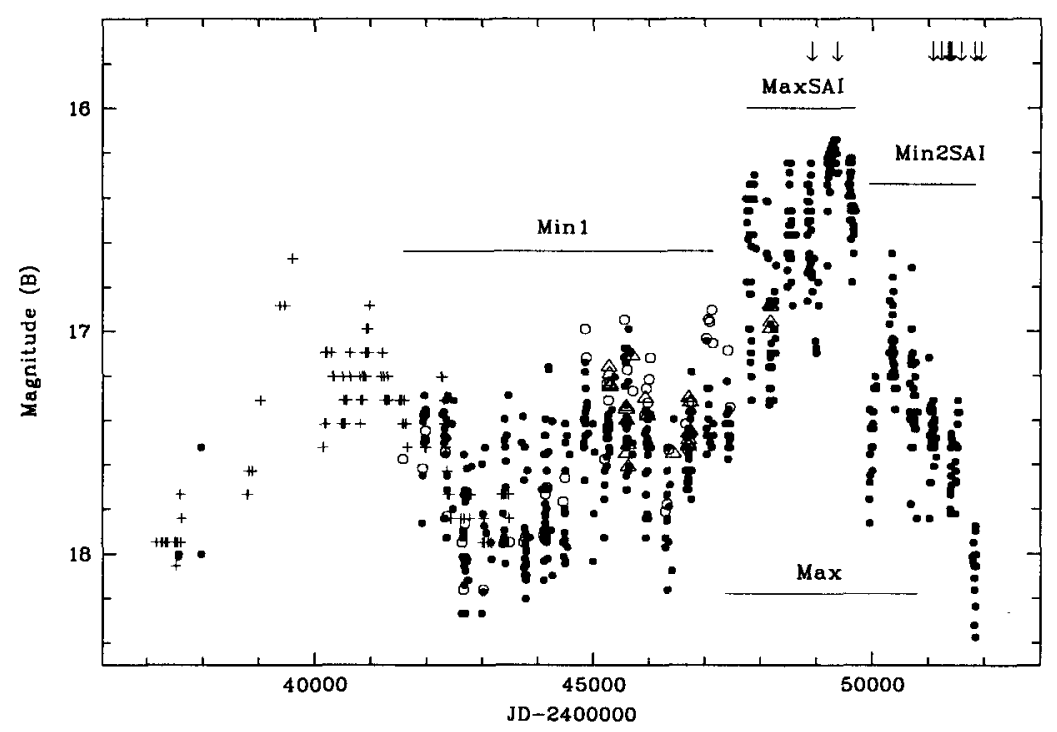

Figure 1. The total light curve of v 532. Filled circles - SAI data, circles - SAI and Baldone Observatory data (Sharov, 1990), crosses Romano's data (Romano, 1978), triangles - Rozhen data (Kurtev et al., 2001) The selected intervals are shown. Dates of spectral observations are marked with vertical arrows.

brightness states. The amplitudes rise when the star becomes brighter. This property of LBV stars is well known (Lamers et al., 1998). A nother known property - longer periods have greater amplitudes - is clearly seen. However, the slope of these dependences decreases when we move from long to short periods, it almost disappears in 20-30-day periods and is reversed for shorter periods. We suggest that the 20-30-day and shorter periods are pulsation periods of $\mathrm{v} 532$. The longer periods may be wind pulsations driven by wind instabilities.

Acknowledgments. O. Sholukhova is grateful to the Scientific and the Local Organizing Committee of the IAU Colloquium 185 for support. This work was supported by RFBR grant N 00-02-16588.

\section{References}

Kurtev, R., et al. 2001, RevMexAA, 37, 57

Lamers, H.J.G.L.M., et al., 1998, A\&A, 335, 605

Romano, G. 1978, A\&A, 67, 291

Sharov, A. 1990, Soviet Astr., 34, 364 